\title{
Computational Models for Social and Technical Interactions
}

\author{
João Gama ${ }^{1}$ Eugénio Oliveira ${ }^{2} \cdot$ Henrique Lopes Cardoso ${ }^{2}$
}

Artificial intelligence promises to revolutionize our lives, changing the way how we work, think and live. We are witnesses of driverless cars, computer-based diagnose systems, office automatization, industry virtualization, etc.

We interact with algorithms for decision support in our daily activities. Typically, these systems used to act in silence. Nowadays, they came to the front pages: DeepBlue won against Kasparov, AlphaGo won the Go world champion, there are robots in Mars, IBM Watson won Jeopardy!, and we are witnessing significant advances in areas such as voice recognition, face recognition, and automatic translation.

Over the last years, a new world of small and heterogeneous devices (mobile phones, PDA, GPS devices, intelligent meters, etc.) have emerged. They are equipped with limited computational and communication power, and have the ability to sense, to communicate and to interact over some communication infrastructure. These large scale distributed systems have in many cases to interact in real-time. This is the advent of virtualized worlds that are the basis of Industry 4.0.

All these scientific developments make us perceive the world as faster and smaller. We have access, in our hands, to real-time and personalized information available $24 \mathrm{~h} /$ day, 7 days/week. There are plenty of innovation opportunities. Nevertheless, there are also weakness points. The world became much more chaotic

João Gama

jgama@fep.up.pt

Eugénio Oliveira

eco@fe.up.pt

Henrique Lopes Cardoso

hlc@fe.up.pt

1 INESC TEC, University of Porto, Porto, Portugal

2 LIAAC, University of Porto, Porto, Portugal 
and risky. Laws and ethics in a virtualized world are not yet defined. Moreover, privacy must be redefined, and trustability might become a strong issue.

In the context of the 18th EPIA Conference on Artificial Intelligence, we have promoted a journal special issue for papers describing recent advances in AI, mostly based on computational models for social and technical interactions. The call for papers referred: This journal issue invites high-quality submissions that present original work in the areas of computational models for understanding, modeling, and facilitating social interaction between people, organizations and systems. Topics covering intelligent socio-technical systems, adaptive and social-aware intelligent systems, evolving social systems, adaptive and reactive intelligent systems, governance mechanisms, organizational learning, social media analysis, social network analysis, social robotics, smart cities, V2V, etc., are welcome.

We received 26 submissions. After a careful and demanding reviewing process, six papers have been selected. All accepted papers have been presented at the 18th EPIA Conference on Artificial Intelligence, held in Porto, 5th-8th September 2017.

Three papers analyze social media data. The paper, TexRep: A Text Mining Framework for Online Reputation Monitoring by Pedro Saleiro, Eduarda Mendes Rodrigues, Carlos Soares, and Eugénio Oliveira, uses unstructured text data from different Web sources for online reputation monitoring. The system collects texts from online media, such as Twitter, and identifies entities of interest and classifies sentiment polarity and intensity. The framework supports multiple data aggregation methods, as well as visualization and modeling techniques that can be used for both descriptive analytics, such as analyze how political polls evolve over time, and predictive analytics, such as predict elections.

The paper, Predicting the Relevance of Social Media Posts Based on Linguistic Features and Journalistic Criteria by Hugo Gonçalo Oliveira, Alexandre Pinto, Álvaro Figueira, and Ana Alves, focuses on the automatic classification of public social text according to its potential relevance, from a journalistic point of view. Authors select a set of posts with several criteria, including the journalistic relevance, assessed by human judges. Using natural language processing tools, the system extracts linguistic features used to learn a classifier. The classifier is used to predict the journalistic relevance of query posts.

The paper, A Framework for Recommendation of Highly Popular News Lacking Social Feedback by Nuno Moniz, Luís Torgo, Magdalini Eirinaki, and Paula Branco, studies the task of predicting news popularity upon their publication, when social feedback is unavailable or scarce. Unlike previous work, authors focus on accurately predicting highly popular news. Such cases are rare, causing known issues for standard prediction models and evaluation metrics. To overcome such issues they propose the use of resampling strategies to bias learners towards these rare cases of highly popular news, and a utility-based framework for evaluating their performance.

The other three papers deal with complex data, from videos and sound to highspeed data streams. The paper, Tensor Based Shot Boundary Detection in Video Streams by Boguslaw Cyganek and Michal Wozniak, presents a method for content change detection of multi-dimensional video signals. Video frames are represented as tensors of order consistent with signal dimensions. 
The paper, Automatic Classification of Impact Sounds with Rejection of Unknown Samples by Joaquim Ferreira da Silva, Sofia Cavaco, and Gabriel Pereira Lopes, presents a method to automatically select the features to be used in discriminating sounds. Given an initial large set of features, the method measures their discriminative power and builds a reduced set of new features which discriminates the sound classes very accurately. This feature selection method is part of the learning phase of a supervised classification approach also proposed here.

The paper, WCDS: A Two-Phase Weightless Neural System for Data Stream Clustering by Douglas O. Cardoso, Felipe M. G. França, and João Gama, presents WCDS, an approach for data streams clustering based on the WiSARD artificial neural network model. This model satisfies all the strict constrains of the data streams computational model. The system presents novel characteristics such as the ability to cluster unbalance data, and new mechanisms to discard outdated data.

This special issue would not have been possible without the help of many people. In particular, we would like to thank the referees for their hard work and timely reviewing of the papers submitted to the special issue.

João Gama received, in 2000, his Ph.D. degree in Computer Science from the Faculty of Sciences of the University of Porto, Portugal. He joined the Faculty of Economy where he holds the position of Associate Professor. He is also a senior researcher and vice-director of LIAAD, a group belonging to INESC TEC. He has worked in several National and European projects on Incremental and Adaptive learning systems, Ubiquitous Knowledge Discovery, Learning from Massive, and Structured Data, etc. He served as CoProgram chair of ECML'2005, DS'2009, ADMA'2009, IDA'2011, ECM-PKDD'2015, DSAA 2017, and EPIA 2017. He served as track chair on Data Streams with ACM SAC from 2007 till 2016. He organized a series of workshops on Knowledge Discovery from Data Streams with ECMLPKDD conferences and Knowledge Discovery from Sensor Data with ACM SIGKDD. He is the author of several books on Data Mining and authored a monograph on Knowledge Discovery from Data Streams. He authored more than 250 peer-reviewed papers in areas related to machine learning, data mining, and data streams. $\mathrm{He}$ is a member of the editorial board of international journals ML, DMKD, TKDE, IDA, NGC, and KAIS. He is a visiting professor at Carlos III University and at Telecom ParisTech.

Eugénio Oliveira is Full Professor at the University of Porto (FEUP) and ex-Director of LIACCArtificial Intelligence and Computer Science Lab at the same University. He is also Director of the Doctoral Program in Informatics Engineering. He was the Event co-chair of the 18th EPIA-Artificial Intelligence Conference. He got his PhD at UNL in Informatics (Artificial Intelligence) (84) and went to IBM/IEC, La Hulpe, as Guest Academic (84-85). He also was research engineer at BBC, near Zurich, in the 1970s. He was awarded the Gulbenkian Prize for Science and Technology in 1984 (ex-aequo). He successfully supervised more than $20 \mathrm{PhDs}$ and has more than 200 indexed publications (no. of citations $=4224$, Hindex=30, i10 index=109). He is co-author of the book "A New Approach for Disruption Management in Airline Operations Control", Springer Verlag. He is or was member of the Editorial Board of prestigious journals like "Autonomous Agents and Multi-Agent Systems", Springer. He was a visitant professor at several universities and schools in Nice, QM-U. London, Utrecht, Madrid, Grenoble, Saint Etiénne, Barcelona. He was project reviewer for the French "Agence Nationale de Recherche", the Spanish "Ministério de Ciencia e Innovación" and the Portuguese "Fundação para a Ciência e Tecnologia". His main research interests are on Distributed Artificial Intelligence, Multi-agent Systems and Text Mining.

Henrique Lopes Cardoso is an Assistant Professor at the Department of Informatics Engineering (DEI) of the Faculty of Engineering of the University of Porto (FEUP). He holds a PhD on Informatics Engineering (University of Porto), and an MSc on Artificial Intelligence and Computation. He is an integrated researcher at the Artificial Intelligence and Computer Science Laboratory (LIACC) of the 
University of Porto. He is a member of the directive board of the Portuguese Association for Artificial Intelligence (APPIA). He coordinates the Argumentation Hub (ARGH), a multidisciplinary and collaborative lab on the topic of Argumentation, within the Media Innovation Labs (UPorto). He is a member of the Follow-up Committee of the Doctoral Program on Informatics Engineering (ProDEI), and a member of the Informatics Department Council. His main research interests are related with Artificial Intelligence, Multi-Agent Systems (MAS), social aspects in MAS (norms, computational trust, incentives, and argumentation), multi-agent systems exploring social coordination (negotiation, cooperation), tools for programing MAS and for simulating agent-based systems, machine learning and text/argumentation mining. He is co-Program Chair of the 18th EPIA Conference on Artificial Intelligence (EPIA 2017). He has participated in several program committees of international conferences and workshops, and has been involved in European research networks (e.g. Agreement Technologies, SINTELNET) and in several research projects (both national and European). 